\title{
Analysis SARS-CoV-2 Genomes of G20 Areas on Phylogeny Tree, t-SNE based on Machine Learning
}

Renyang Liu

Yunnan University

Mu Qiao

Yunnan University

Alima

Yunnan University

Jeffrey Zheng ( $\nabla$ conjugatelogic@yahoo.com )

Yunnan University

Wei Zhou

Yunnan University

\section{Research Article}

Keywords: COVID-19, SARS-CoV-2, feature extraction, machine learning, gene sequence, t-SNE, phylogenetic tree

Posted Date: January 11th, 2021

DOl: https://doi.org/10.21203/rs.3.rs-74633/v2

License: (c) (i) This work is licensed under a Creative Commons Attribution 4.0 International License.

Read Full License 


\title{
Analysis SARS-CoV-2 Genomes of G20 Areas on Phylogeny Tree, t-SNE based on Machine Learning
}

\author{
Renyang Liu, Mu Qiao, Alima, Jeffrey Zheng, Wei Zhou
}

\begin{abstract}
The new coronavirus disease (COVID-19) broke out earlier in Wuhan, and the plague spread rapidly from multiple resources of different countries. COVID-19 has caused millions of diagnosed people worldwide, causing many deaths and posing a severe threat to public health in countries around the world. Facing this urgent situation, in-depth research on the emerging SARS-CoV-2 to understand the related pathogenic mechanism and epidemiological characteristics is urgent. This type of activity would be useful to determine its origin to formulate effective prevention and treatment strategies for affected patients.

This paper adopts t-SNE based on machine learning to draw a phylogenetic tree from collected genomic sequences to analyze G20 countries' samples. The phylogenetic tree of the generating mechanism was described, and intermediate results were illustrated. The results of this research showed that viruses in many countries have similar or similar relationships among the gene sequences.
\end{abstract}

Keyword COVID-19, SARS-CoV-2, feature extraction, machine learning, gene sequence, $\mathrm{t}$-SNE, phylogenetic tree

Renyang Liu

Yunnan University,Kunming e-mail: liurenyang@mail.ynu.edu.cn

Mu Qiao

Yunnan University, Kunming e-mail: qiaomu@mail.ynu.edu.cn

Alima

Yunnan University, Kunming e-mail: 843450368 @qq. com

Jeffrey Zheng

Yunnan University, Kunming e-mail: conjugatelogic@yahoo.com

Wei Zhou

Yunnan University, Kunming e-mail: zwei@ynu.edu.cn

This work was supported by the NSFC (61762089, 61663047, 61863036, 61762092, 62041213), the Science and Technology Innovation Team Project of Yunnan Province (2017HC012), and the Key Project on Electric Information and Next Generation IT Technology of Yunnan (2018ZI002) 


\section{Introduction}

Coronavirus belongs to the Nestoviruses, Coronaviridae, and Coronavirus genera. It is a type of RNA virus with an envelope and a linear single-stranded genome. It is a large class of viruses that widely exist in nature. Certain coronaviruses can infect humans and cause diseases, such as Middle East respiratory syndrome (MERS) and severe acute respiratory syndrome (SARS), whose symptoms can range from a common cold to severe lung infections.

The coronavirus outbroken earlier in Wuhan in December 2019 is a virus strain that has not been previously discovered in humans and was named by the WHO as the 2019 novel coronavirus "2019-nCoV" (2019 novel Coronavirus). On February 11, 2020, the virus was named "SARS-CoV-2" (Severe Acute Respiratory Syndrome Coronavirus 2) by the Coronavirus Study Group (CSG) of the International Committee on Taxonomy of Viruses. At the same time, the disease caused by the virus infection was named "COVID-19" (Corona Virus Disease 2019) by WHO. 2019-nCoV is a new member of the severe acute respiratory syndrome coronavirus family (SARS-CoV) and is labeled SARS-CoV-2 [7].

In the following days, from Europe to North America, its fatal impact is threatening the entire world. According to the World Health Organization (WHO) latest update data, more than 2 million people have been diagnosed in more than 200 countries and regions across the country. According to $\mathrm{WHO}^{1}$, in these cases, approximately 200,000 confirmed cases died. The new coronavirus (2019-nCoV) meets the definition of all epidemics actively. COVID-19 manifests as fever, sore throat and pneumonia and accompanying severe acute respiratory distress symptoms [6].

Although researchers worldwide have invested considerable time and energy in this work, the relationship of COVID-19 in different regions has not yet formed a conclusion.

Researchers, however, have proposed many classification algorithms based on machine learning. Most of these algorithms are unsupervised algorithms that are very suitable for the classification of viruses in various countries to obtain a rough classification of viruses in various countries. Therefore, in this study, we tried t-SNE algorithms in machine learning to cluster the gene sequences of G20 countries and construct a phylogenetic tree to show virus evolution. From the experimental results, we can see that the virus sequences in these countries can be divided into various major categories, that is, the sources of viruses in countries classified by machine learning in the same category may be the same.

Here, we describe our work as follows:

- Exploring the different $\mathrm{k}$ of $\mathrm{k}-\mathrm{mers}$ as virus characteristics,

- We use a variety of machine learning-based algorithms t-SNE to cluster the G20 countries' new coronavirus gene sequences to explore the relationship between viruses in each country

- Attempt to show the connection and mutation of viruses among countries through the phylogenetic tree.

\footnotetext{
1 https://covid19.who.int/
} 
The rest of the paper is composed as follows: Materials and Methods introduce our work's bases. Next, the experiments and discussion are included. Finally, our research conclusions are proved.

\section{Materials and Methods}

In this section, we will give a detailed introduction to the materials and methods used.

\section{Datasets}

All datasets used in this work were from various open-source genomic banks $\mathrm{CNCB}^{2}$ and GISAID $^{3}$, and we cleaned the data. The virus information is shown in Table.1:

Table 1: aThe information about virus' gene sequence

\begin{tabular}{lllll}
\hline No. Name & Id & \multicolumn{3}{c}{ Collected Date Locality } \\
\hline 1 & Australia.fasta & EPI_ISL_420006 $2020-03-24$ & Australia \\
2 & Belgium.fasta & EPI_ISL_420432 2020-03-23 & Belgium \\
3 & Brazil.fasta & EPI_ISL_427306 2020-03-10 & Brazil \\
4 & Canada.fasta & EPI_ISL_413014 2020-01-25 & Canada \\
5 & Chile.fasta & EPI_ISL_414580 2020-03-05 & Chile \\
6 & China.fasta & EPI_ISL_414692 2020-02-25 & China \\
7 & England.fasta & EPI_ISL_414500 2020-03-04 & England \\
8 & France.fasta & EPI_ISL_414500 2020-03-23 & France \\
9 & Germany.fasta & EPI_ISL_414521 2020-03-02 & Germany \\
10 & India.fasta & EPI_ISL_424365 2020-03-17 & India \\
11 & Italy.fasta & EPI_ISL_419254 2020-03-23 & Italy \\
12 & Japan.fasta & EPI_ISL_419307 2020-03-20 & Japan \\
13 & Mexico.fasta & EPI_ISL_424673 2020-03-12 & Mexico \\
14 & Russia.fasta & EPI_ISL_420080 2020-03-18 & Russia \\
15 & SaudiArabia.fasta & EPI_ISL_416522 2020-03-10 & SaudiArabia \\
16 & Singapore.fasta & EPI_ISL_420111 2020-03-12 & Singapore \\
17 & SouthAfrica.fasta & EPI_ISL_421575 2020-04-01 & SouthAfrica \\
18 & SouthKorea.fasta & EPI_ISL_413516 2020-02-27 & SouthKorea \\
19 & Turkey.fasta & EPI_ISL_424366 2020-03-17 & Turkey \\
20 & USA.fasta & EPI_ISL_424353 2020-04-02 & USA
\end{tabular}

\footnotetext{
2 https://bigd.big.ac.cn/ncov/release_genome

${ }^{3}$ https://www.gisaid.org/epiflu-applications/next-hcov-19-app/
} 
$t-S N E$

$\mathrm{t}$-SNE (t-distributed stochastic neighbor embedding) [3] is a machine learning algorithm for dimensionality reduction. Laurens van der Maaten proposed it, and Geoffrey Hinton in 2008, based on SNE [4], proposed it. t-SNE is a nonlinear dimensionality reduction algorithm that is very suitable for high-dimensional data reduction to 2 or 3 dimensions for visualization. In addition, it has meaning when the data are marked, which can clearly show the input data's clustering status. The main idea is to use conditional probabilities to represent the distance of high-dimensional distribution points and low-dimensional distribution points. As long as the conditional probabilities of the two are very close (training with relative entropy, so labels are needed), it means that the points of the high-dimensional distribution have been mapped to the low-dimensional distribution.

In this article, the t-SNE algorithm clusters viral gene sequences in the following two steps:

- Extract the feature vectors of virus genes from G20 countries

- Using the t-SNE algorithm to cluster the extracted feature vectors

\section{Feature extraction}

The gene sequence is composed of four essential elements: \{"A", "T", "C", "G" $\}$. The length of each COVID-19 virus gene sequence is approximately 30,000. To cluster the G20's virus gene sequences with t-SNE, we first extracted the corresponding features of each sequence.

Before that, we first need to know the concept of a proper noun, mer (monomeric unit, mer), which means monomer unit in molecular biology. Units commonly used in nucleic acid sequences represent nt or bp. For example, 100 mer DNA represents a single-strand length of $100 \mathrm{nt}$ or a double-strand length of $100 \mathrm{bp}$. The k-mer [5] refers to dividing the nucleic acid sequence into a string containing $k$ bases, that is, iteratively selecting a sequence of length $k$ bases from a contiguous nucleic acid sequence. If the length of the nucleic acid sequence is $L, \mathrm{k}$-mer If the length is $k$, then $L-K+1 \mathrm{k}$-mers can be obtained.

We use the number of k-mers as the characteristics of our gene sequence in our work, but unlike other k-mers methods, we will count the repeated k-mer only once. We need to explore which k-mers are the most important components of gene sequences. Therefore, we designed an experiment to explore the relationship between k-mers of different lengths and viral gene sequences. Finally, we found that the length $k$ satisfying $5 \leq k \leq 40$ is more appropriate. 


\section{Clustering use t-SNE}

After extracting the gene feature, we use the number of mer types minus the average and average of the corresponding mer number as the feature vector of each virus gene. Then, the t-SNE algorithm is used to cluster and display the viral sequences of $\mathrm{G} 20$ countries.

\section{Phylogenetic tree}

A phylogenetic tree [1] or evolutionary tree is a kind of tree structure diagram commonly used to express the genealogical relationship of species. At the molecular level, the distance between kinship is usually expressed by differences in DNA (or protein) sequences.

There are many ways to build a phylogenetic tree, and we use the distance method in our work. The distance-dependent method means that the two sequences' evolutionary distances determined the topological shape of the phylogenetic tree. The length of the clade branch represents the evolutionary distance.

$$
d(x, y)=\sqrt{\left(x_{1}-y_{x}\right)^{2}+\left(x_{2}-y_{2}\right)^{2}+\ldots+\left(x_{n}-y_{n}\right)^{2}}=\sqrt{\sum_{i=1}^{n}\left(x_{i}-y_{i}\right)^{2}}
$$

Specifically, we use UPGMA (unweighted pair group method with arithmetic mean) based on the distance method in our work, where distance refers to Euclidean distance (Eq. 1) [2]. The detailed steps are as follows:

- We first generate a "distance matrix" by comparing two gene sequences and then calculating each pair of sequences' gene distances. In short, the number of two sequences that do not match (of course, the actual calculation is more important than this Much trouble);

- Then, the pairwise alignment distance matrix is used to estimate the two sequences with the shortest distance. These two sequences form the two clades of the evolutionary tree. Then, the distance matrix between these two alignments restarts to find the two closest sequences. However, unlike before, the two most similar sequences are connected to the tree by one node. And so on until the end.

- Draw the tree according to the distance with Biopython ${ }^{4}$.

\footnotetext{
${ }^{4}$ https://github.com/biopython/biopython.github.io
} 


\section{Experiments and Discussion}

\section{Find the suitable $k$ of $k$-mers}

The results in Fig. 1 show that when the k of k-mers of the subsequence is too short, the number of mers is particularly poor. For example, when $\mathrm{k}$ equals 1 , there are only four types of mers. The $\mathrm{k}$ is too large; however, the types of mers are close to the length of the gene sequence. For example, when $\mathrm{k}$ equals 100 , the number of mers is close to 3000 . That is, when $\mathrm{k}$ is less than or greater than a certain length, it has almost no effect on the division of different genes for each country, and all the polylines will tend to be stable. Moreover, to observe more clearly, we subtracted the average of the number of categories corresponding to each mer. The result is shown in Fig. 2.

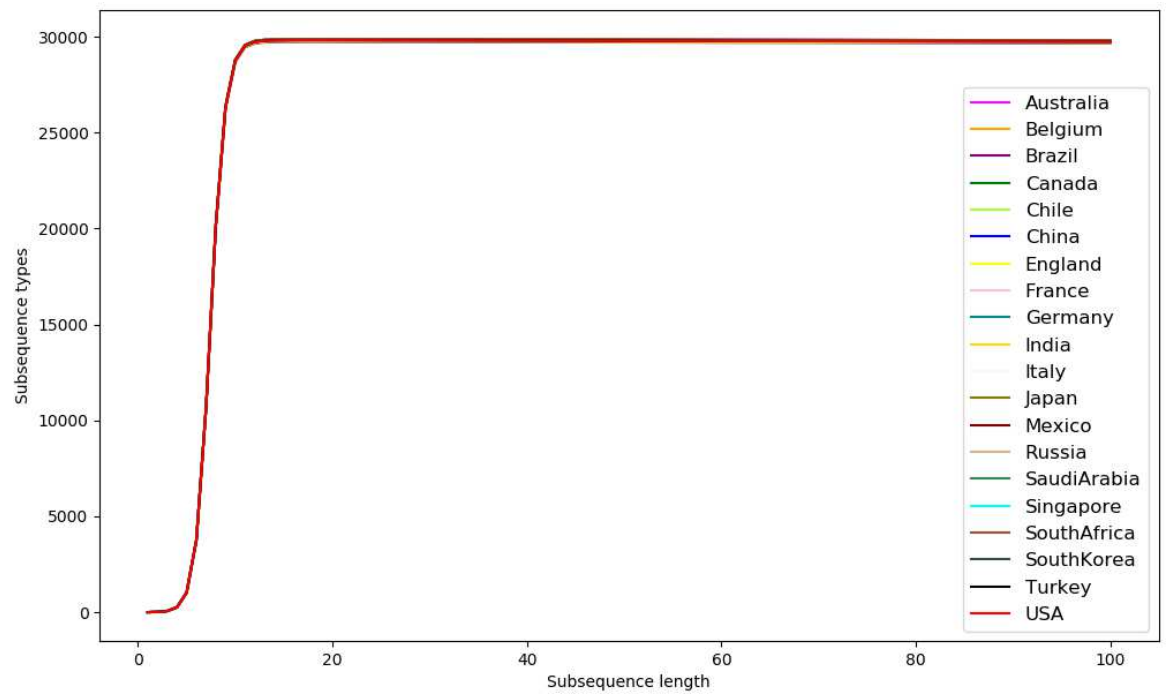

Fig. 1: The relationship between different $\mathrm{k}$ and mer types. The abscissa is the length $\mathrm{k}$ of mer, and the ordinate is the various numbers of the mer. 


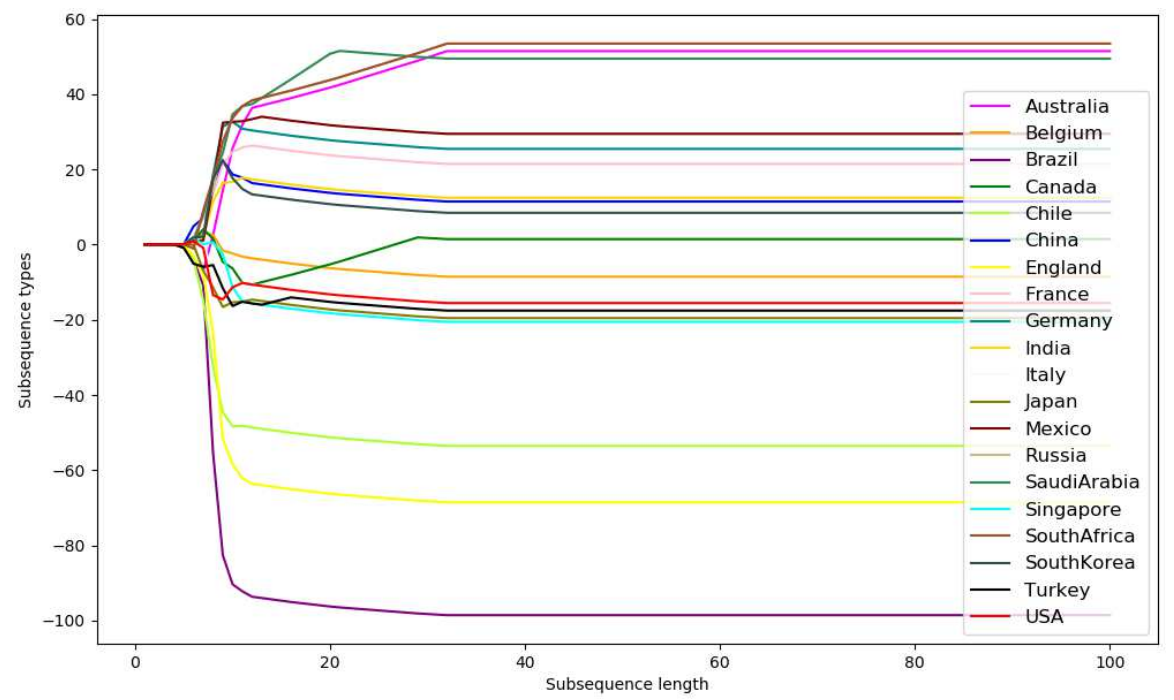

Fig. 2: The relationship between different $\mathrm{k}$ and the mer types after subtracting the average value. The abscissa is the length $\mathrm{k}$ of mer, and the ordinate is the various numbers of the mer.

According to the experimental results, we can see that when the length satisfies $5 \sim 40$, where $k$ is the length of the mers, the types of mers become more evident, so we use $5 \leq k \leq 40$ as the length of the mer to extract features of the virus sequence and carry out the next exploration experiment.

\section{Clustering with t-SNE}

The clustering results with t-SNE of the viral gene sequences of G20 countries are shown in Fig. 3,4, Fig. 3,4. We can see that the t-SNE algorithm clustered the viral gene sequences into at least eight major categories. For example, it clusters France, Germany, and Mexico into one category, which means that their virus sources may be the same. Then, the United States, Singapore, Belgium, and Turkey can cluster into another. This phenomenon indicates that their virus source may be the same. Japan's virus gene sequence and other countries have not been clustered but have become a cluster alone, indicating that Japan's virus source may be different from other G20 countries. 


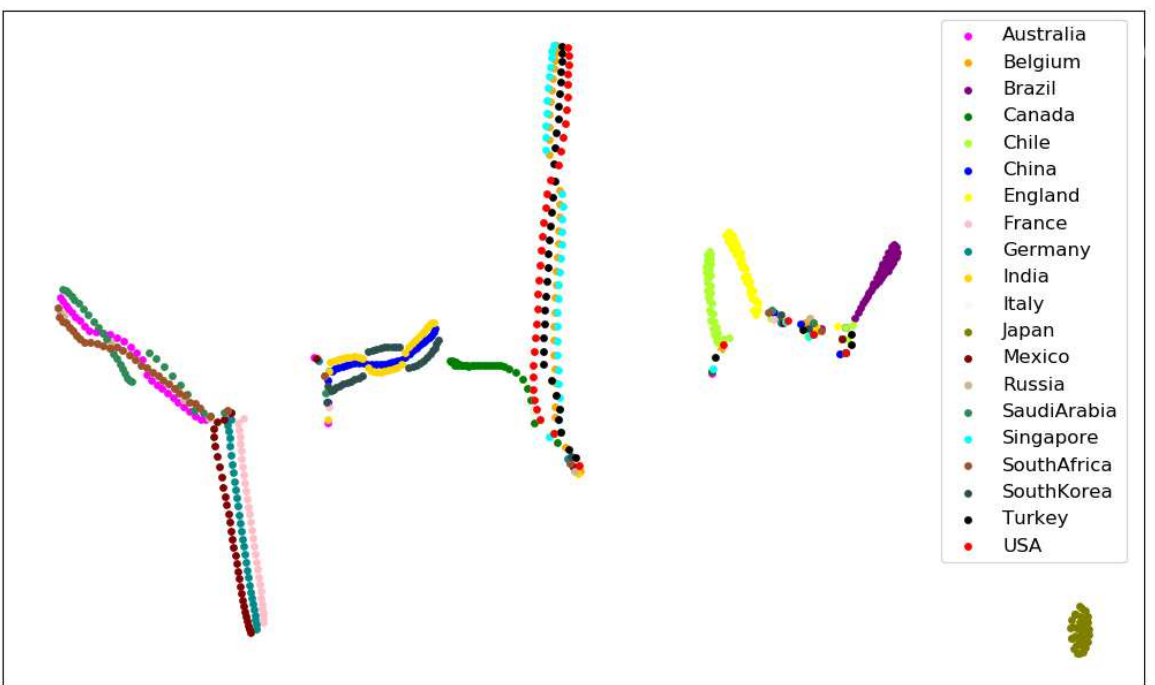

Fig. 3: The clustering results by minus the minimum of corresponding subsequence types.

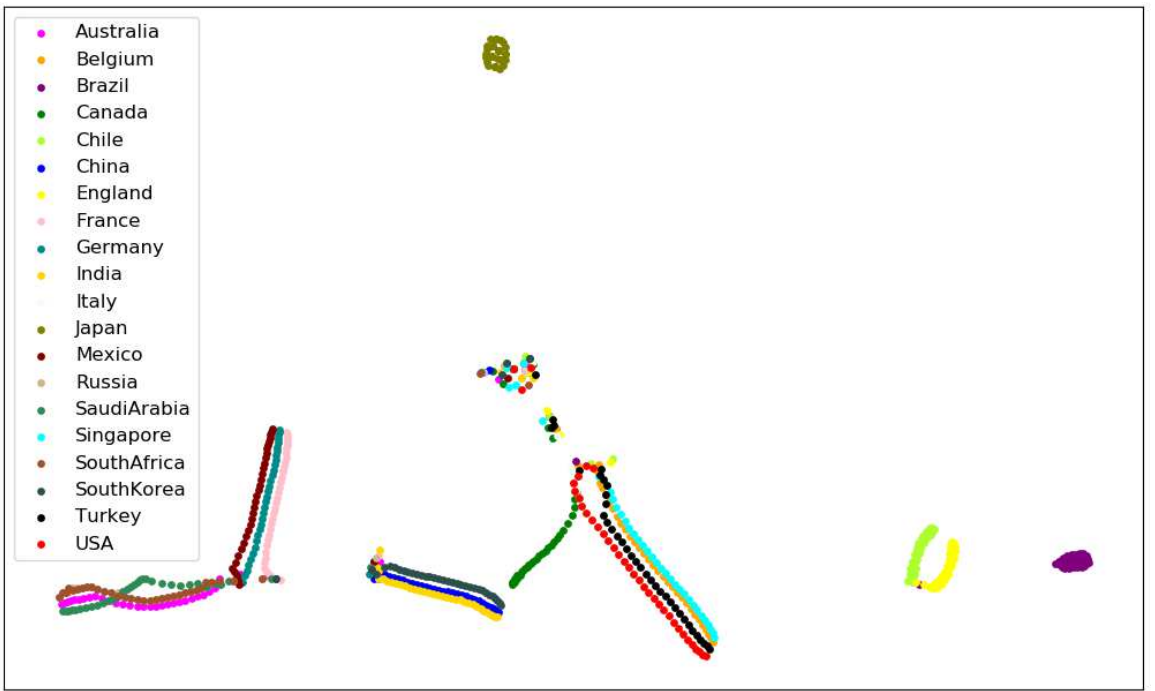

Fig. 4: The clustering results by subtracting the average of the corresponding subsequence types. 


\section{The G20 phylogenetic tree}

Fig. 5 is a phylogenetic tree of virus sequences in G20 countries, which shows the evolution of viruses in G20 countries. It is worth mentioning that no matter how we disturb the input data's order, the results we obtain are the same. This result shows that this phylogenetic tree is relatively stable and can represent the evolution of the gene sequences of G20 countries.

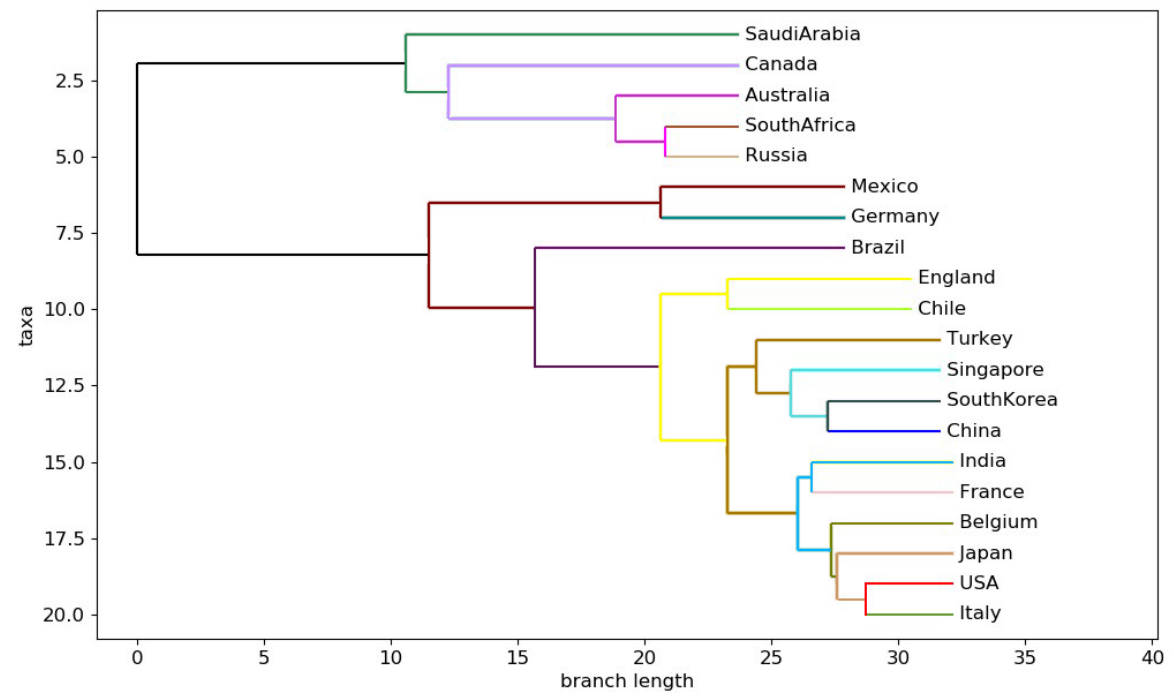

Fig. 5: Phylogenetic tree of viral gene sequences in G20 countries.

\section{Conclusion}

This paper analyzes the gene sequence of COVID-19 in G20 countries and performs many experiments. First, we propose to use a method based on k-mers to extract the features of each virus sequence, select a suitable range of $\mathrm{k}$, and use the mers types as the feature vector of each virus gene. Then, we cluster the virus sequences of various countries by using the machine learning algorithm t-SNE. Finally, we draw the phylogenetic tree of viruses in the G20 countries with subsequences as virus characteristics. Our work found that the viruses of the G20 countries can be roughly divided into eight categories. Furthermore, the phylogenetic tree shows that each country's genetic viruses have a common source and have their characteristics. 


\section{Conflict Interest}

No confilct of interest has claimed.

\section{References}

1. John P. Archer and David L. Robertson. CTree: comparison of clusters between phylogenetic trees made easy. Bioinform., 23(21):2952-2953, 2007.

2. Bruce L. Golden and Michael O. Ball. Shortest paths with euclidean distances: An explanatory model. Networks, 8(4):297-314, 1978.

3. G. E. Hinton. Visualizing high-dimensional data using t-sne. Journal of Machine Learning Research, 9(2):2579-2605, 2008.

4. Geoffrey E. Hinton and Sam T. Roweis. Stochastic neighbor embedding. In Suzanna Becker, Sebastian Thrun, and Klaus Obermayer, editors, Advances in Neural Information Processing Systems 15 [Neural Information Processing Systems, NIPS 2002, December 9-14, 2002, Vancouver, British Columbia, Canada], pages 833-840. MIT Press, 2002.

5. Pavel A. Pevzner, Haixu Tang, and Michael S. Waterman. An eulerian path approach to dna fragment assembly. Proceedings of the National Academy of Sciences of the United States of America, 98(17):9748-9753, 2001.

6. Samar Salman and Mohamed Salem. Routine childhood immunization may protect against covid-19. Medical Hypotheses, page 109689, 032020.

7. Sheng Zhang, Meng Diao, Liwei Duan, Zhaofen Lin, and Dechang Chen. The novel coronavirus (sars-cov-2) infections in china: prevention, control and challenges. Intensive Care Medicine, 46, 032020. 
Figures

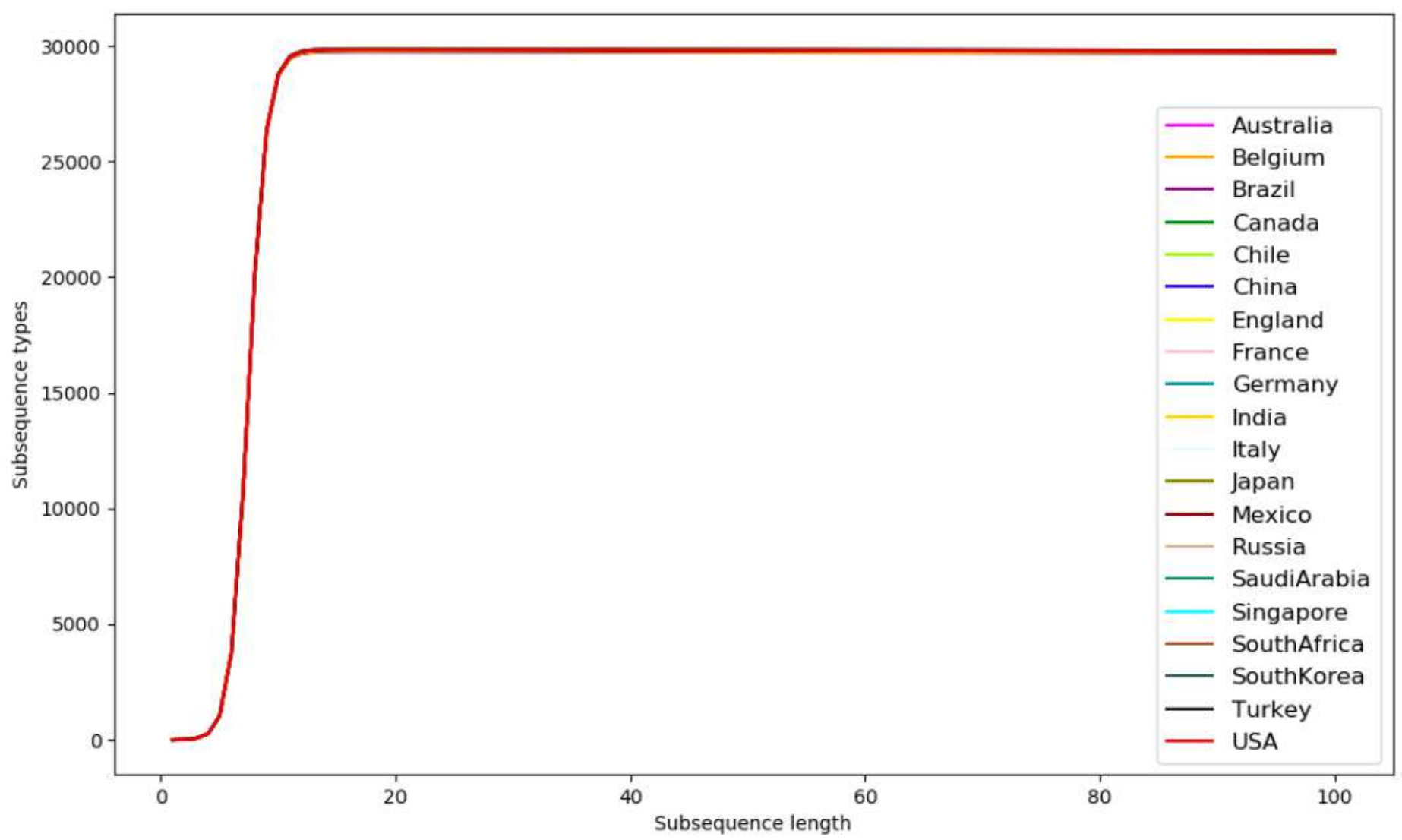

Figure 1

The relationship between different $\mathrm{k}$ and mer types. The abscissa is the length $\mathrm{k}$ of mer, and the ordinate is the various numbers of the mer. 


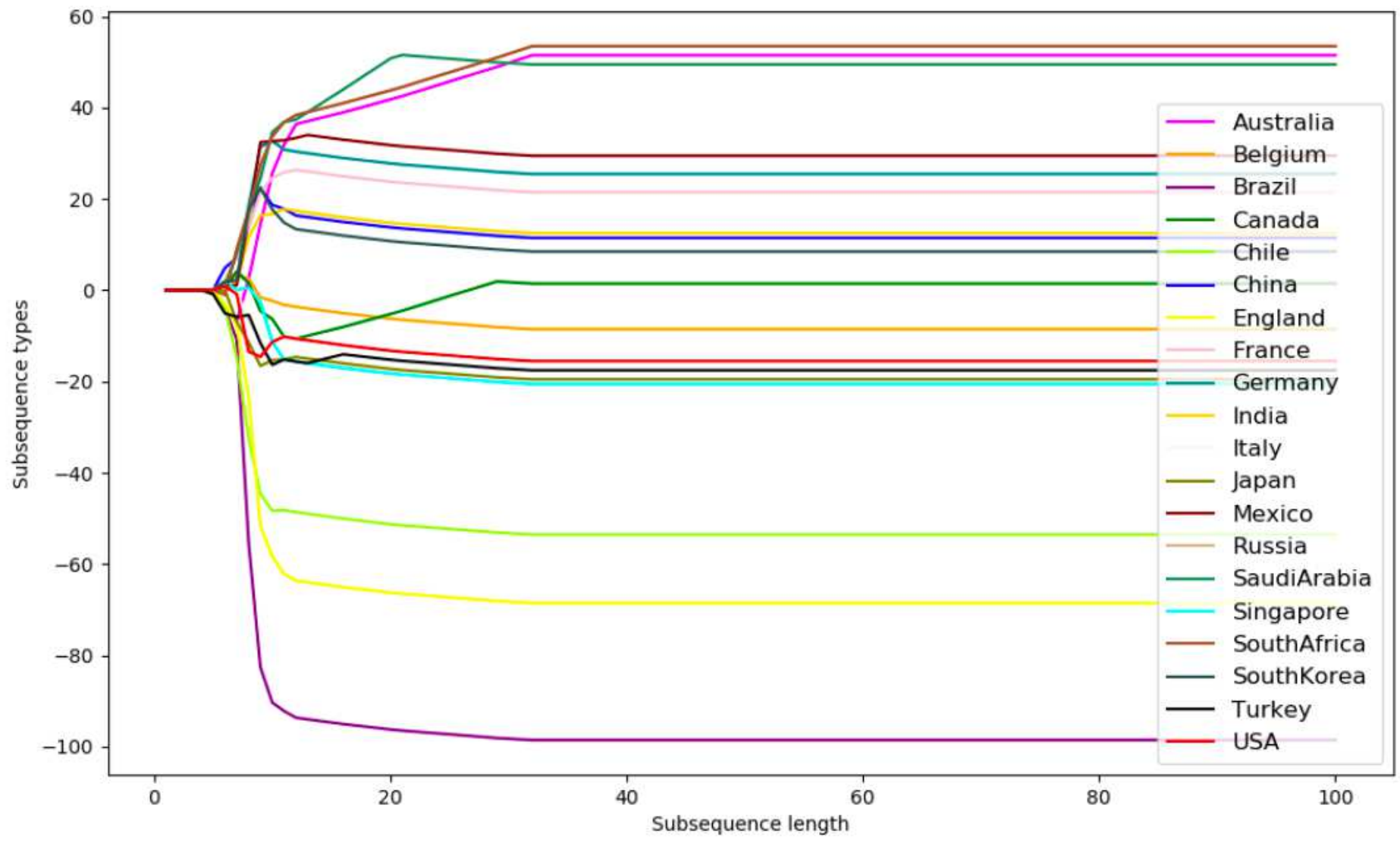

Figure 2

The relationship between different $k$ and the mer types after subtracting the average value. The abscissa is the length $\mathrm{k}$ of mer, and the ordinate is the various numbers of the mer. 


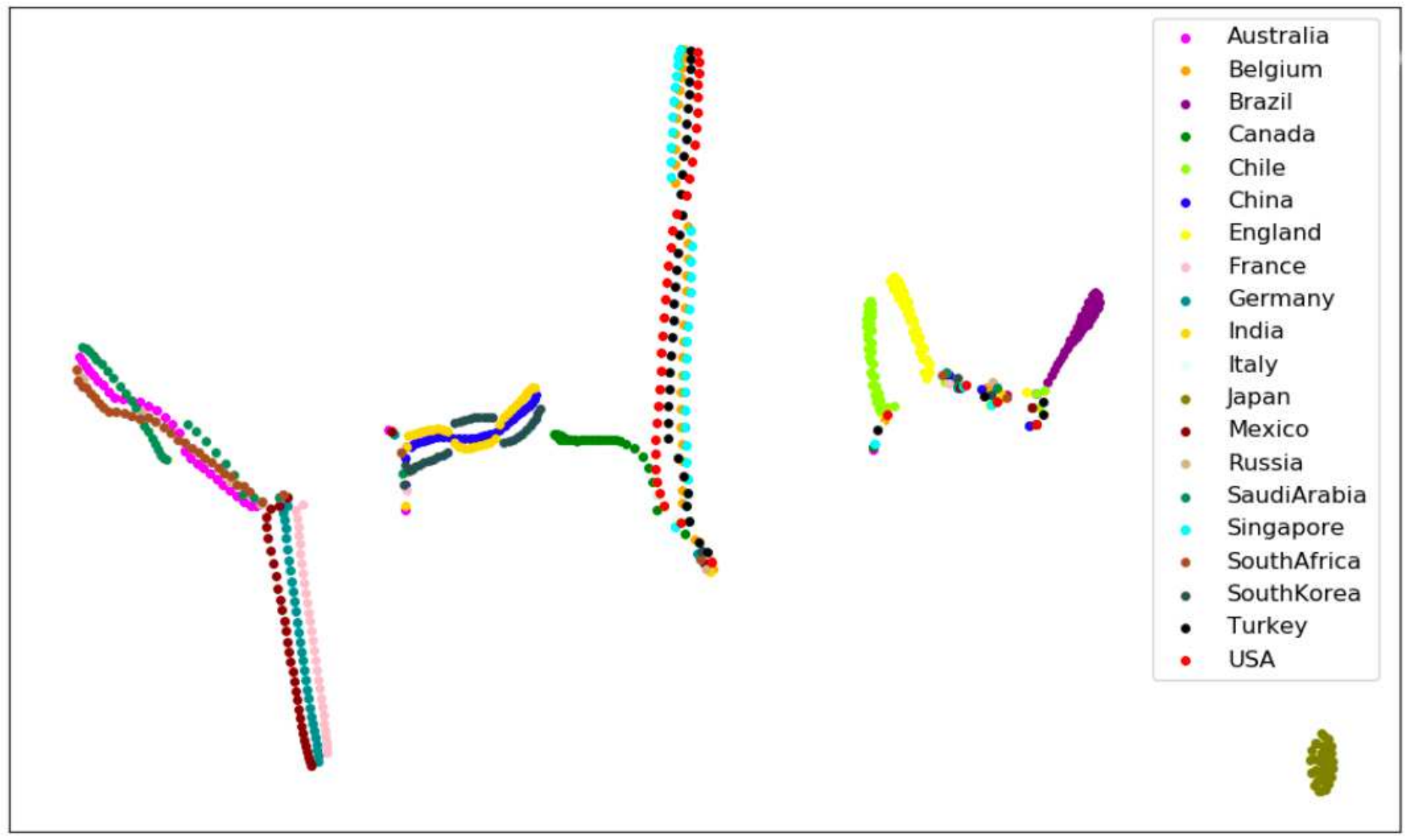

Figure 3

The clustering results by minus the minimum of corresponding subsequence types. 


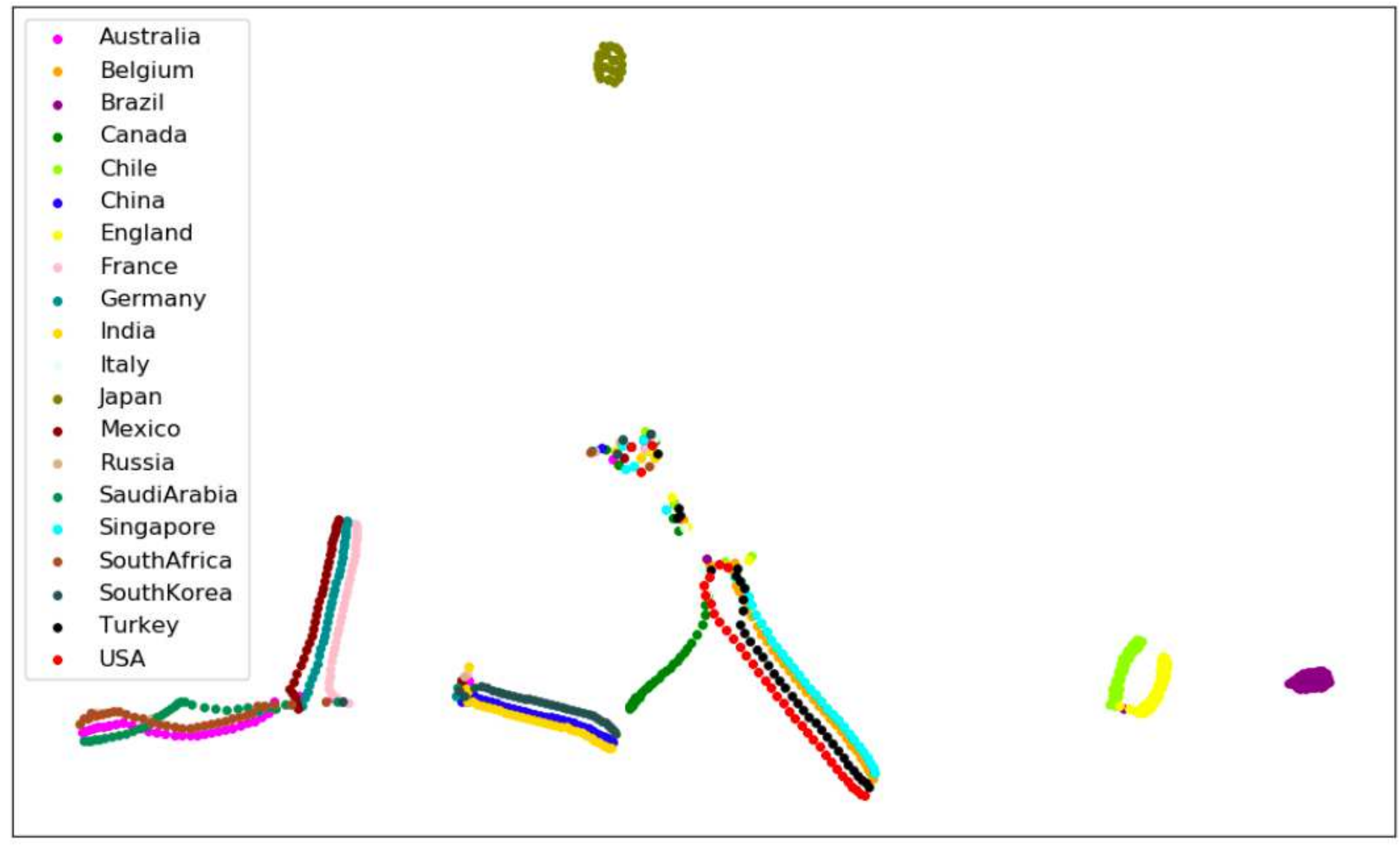

Figure 4

The clustering results by subtracting the average of the corresponding subsequence types. 


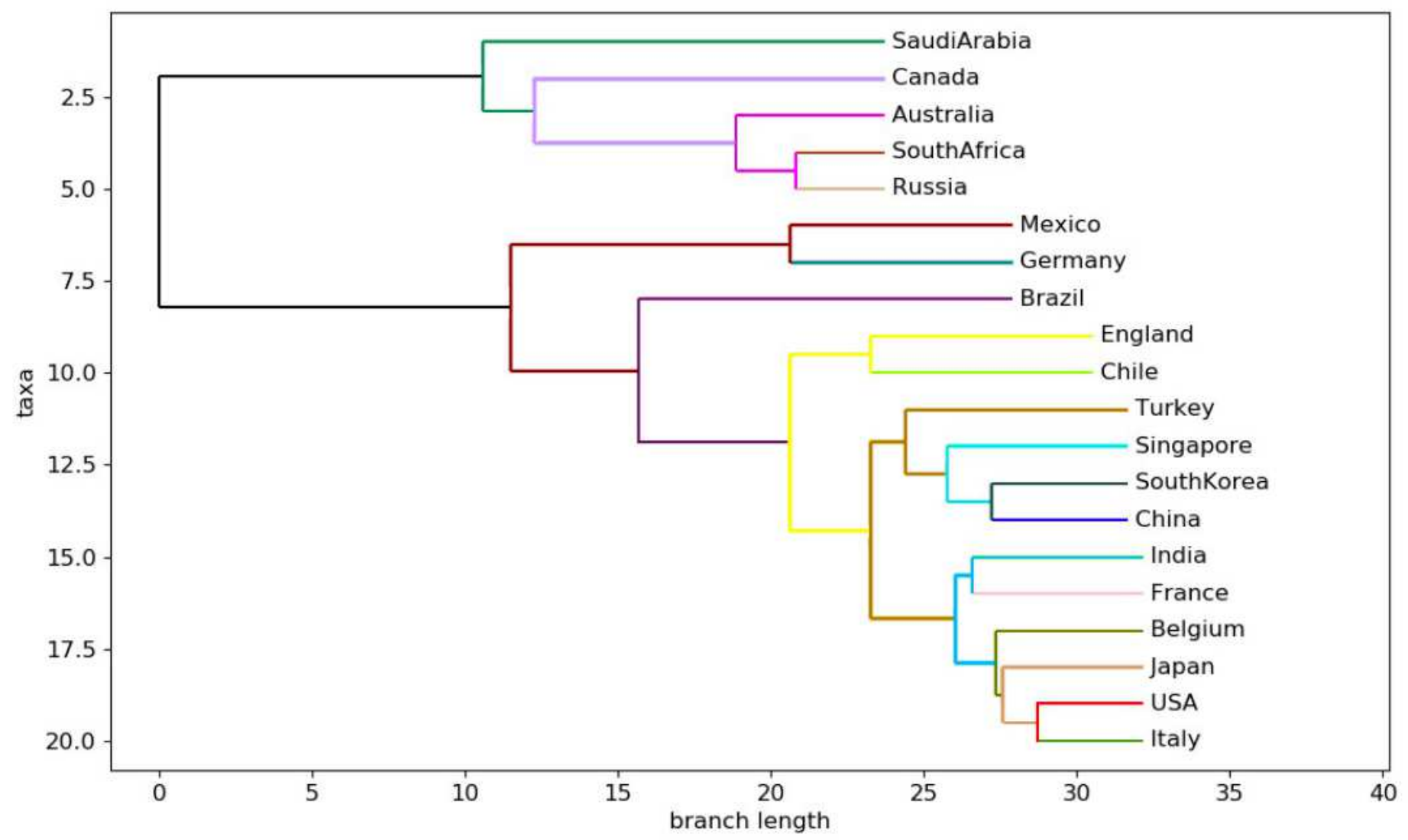

Figure 5

Phylogenetic tree of viral gene sequences in $\mathrm{G} 20$ countries. 